\title{
Maximum Degree Energy of Certain Mesh Derived Networks
}

\section{Rajesh Kanna M.R}

Asst. Professor, Postgraduate Department of Mathematics, Maharani's Science College for Women,

Mysore- 570005.

\author{
Shashi R \\ Lecturer, \\ Department of Computer Science, \\ Yuvaraja's College, Mysore-570005.
}

\author{
Dharmendra B.N \\ Asst. Professor, \\ Maharani's Science College for Women, \\ Mysore-570005.
} Postgraduate Department of Mathematics,

\begin{abstract}
In this paper, we compute the maximum degree energy of grid, cylinder, torus, extended grid networks using mat lab.
\end{abstract}

\section{Keywords}

Grid, Cylinder, Torus, Extended grid, Maximum degree matrix, Maximum degree energy.

\section{INTRODUCTION}

The concept of energy of a graph was introduced by I. Gutman [6] in the year 1978. Let $G$ be a graph with $n$ vertices $\{v 1, v 2, \ldots, v n\}$ and $m$ edges. Let $A=(a i j)$ be the adjacency matrix of the graph. The eigenvalues $\lambda 1, \lambda 2, \ldots$, $\lambda \mathrm{n}$ of $\mathrm{A}$, assumed in non increasing order, are the eigenvalues of the graph G. As A is real symmetric, the eigenvalues of $G$ are real with sum equal to zero. The energy $E(G)$ of $G$, is defined to be the sum of the absolute values of the eigenvalues of $\mathrm{G}$.

$$
E(G)=\sum_{i=1}^{n}\left|\lambda_{\mathrm{i}}\right|
$$

For details on the mathematical aspects of theory of graph energy see the reviews [7], papers [4, 5, 9] and the references cited there in. The basic properties including various upper and lower bounds for energy of a graph have been established in $[11,12]$ and it has found remarkable chemical applications in the molecular orbital theory of conjugated molecules $[10,8]$. Further studies on minimum covering energy, maximum degree energy, minimum dominating energies can be found in $[1,2,13]$ and the references cited there in.

Prof. C. Adiga et al. [2] have defined maximum degree energy of a graph in the year 2009. Recently Bharathi Rajan et al. [3] calculated Laplacian energy of certain mesh derived networks. Motivated by these papers we computed maximum degree energy of certain mesh derived networks. In this paper we are interested in studying mathematical aspects of maximum degree energy of a graph. The applications of maximum degree energy in other branches of science have to be investigated. This may be of great relevance in studying structural properties of these networks in a new angle.

2. MAXIMUM DEGREE ENERGY [2]

Let $G$ be a simple graph with $n$ vertices $v_{1}, v_{2}, \ldots, v_{n}$ and let $d_{i}$ be the degree of $v_{i}, i=1,2, \ldots, n$. Define,

$d_{i j}=\left\{\begin{array}{cc}\max \left(d_{i}, d_{j}\right) & \text { if } v_{i} \text { and } v_{j} \text { are adjacent }, \\ 0 & \text { otherwise } .\end{array}\right.$

Then the $n \times n$ matrix $M(G)=\left[d_{i j}\right]$ is called the maximum degree matrix of $G$. The characteristic polynomial of the maximum degree matrix $M(G)$ is defined by

$$
\begin{aligned}
\varphi(G ; \mu) & =\operatorname{det}(\mu I-M(G)) \\
& =\mu^{n}+c_{1} \mu^{n-1}+c_{2} \mu^{n-2}+\ldots+c_{n},
\end{aligned}
$$

where $I$ is the unit matrix of order $n$. The roots $\mu_{1}, \mu_{2}, \ldots, \mu_{n}$ assumed in non increasing order of $\varphi(G ; \mu)=0$ are the maximum degree eigenvalues of $G$. The maximum degree energy of a graph $G$ is defined as

$$
E_{M}(G)=\sum_{i=1}^{n}\left|\mu_{\mathrm{i}}\right|
$$

Since $M(G)$ is a real symmetric matrix with zero trace, these maximum degree eigenvalues are real with sum equal to zero.

\section{MAXIMUM DEGREE ENERGY OF} SOME STANDARD GRAPHS

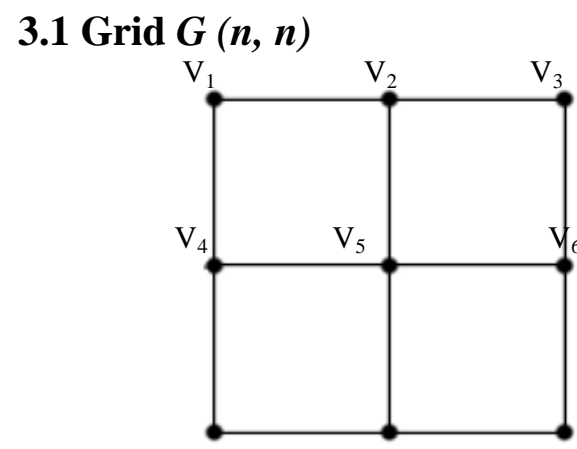




\section{$\begin{array}{lll}\mathrm{V}_{7} & \mathrm{~V}_{8} & \mathrm{~V}_{9}\end{array}$}

Figure 1: Grid $(3,3)$

The maximum degree adjacency matrix of $3 \times 3$ grid is given by

$$
\begin{aligned}
& \mathrm{A}=\left(\begin{array}{lll}
B & D & O \\
D & C & D \\
O & D & B
\end{array}\right) \text { where } \mathrm{B}=\left(\begin{array}{lll}
0 & 3 & 0 \\
3 & 0 & 3 \\
0 & 3 & 0
\end{array}\right), \\
& \mathrm{C}=\left(\begin{array}{lll}
0 & 4 & 0 \\
4 & 0 & 4 \\
0 & 4 & 0
\end{array}\right), \quad \mathrm{D}=\left(\begin{array}{lll}
3 & 0 & 0 \\
0 & 4 & 0 \\
0 & 0 & 3
\end{array}\right) \text { and }
\end{aligned}
$$

$\mathrm{O}$ is the zero matrix of size $3 \times 3$.

In general the maximum degree adjacency matrix of $\mathrm{G}(\mathrm{n}, \mathrm{n})$ for $n \geq 3$ can be written as

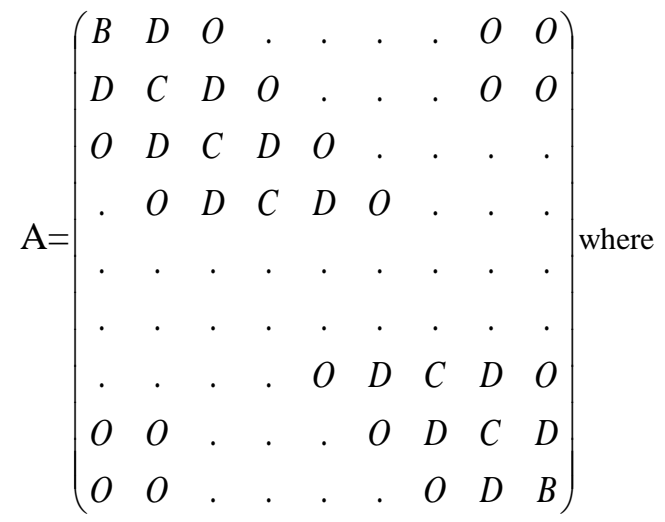

$$
\mathrm{B}=\left(\begin{array}{ccccccccc}
0 & 3 & 0 & . & . & . & . & 0 & 0 \\
3 & 0 & 3 & 0 & . & . & . & 0 & 0 \\
0 & 3 & 0 & 3 & 0 & . & . & . & . \\
. & 0 & 3 & 0 & 3 & 0 & . & . & . \\
. & . & . & . & . & . & . & . & . \\
. & . & . & . & . & . & . & . & . \\
. & . & . & . & 0 & 3 & 0 & 3 & 0 \\
0 & 0 & . & . & . & 0 & 3 & 0 & 3 \\
0 & 0 & . & . & . & . & 0 & 3 & 0
\end{array}\right)
$$

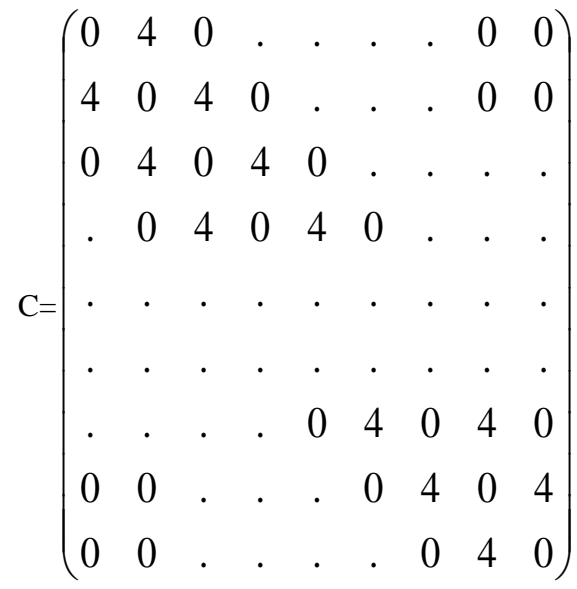

$\mathrm{D}=\left(\begin{array}{ccccccccc}3 & 0 & 0 & . & . & . & . & 0 & 0 \\ 0 & 4 & 0 & 0 & . & . & . & 0 & 0 \\ 0 & 0 & 4 & 0 & 0 & . & . & . & . \\ . & 0 & 0 & 4 & 0 & 0 & . & . & . \\ . & . & . & . & . & . & . & . & . \\ . & . & . & . & . & . & . & . & . \\ . & . & . & . & 0 & 0 & 4 & 0 & 0 \\ 0 & 0 & . & . & . & 0 & 0 & 4 & 0 \\ 0 & 0 & . & . & . & . & 0 & 0 & 3\end{array}\right)$

and $\mathrm{O}$ is the zero matrix of size $\mathrm{n} \times \mathrm{n}$.

The following MATLAB program generates the maximum degree energy of a $\operatorname{Grid}(n, n)$ for $n \geq 3$.

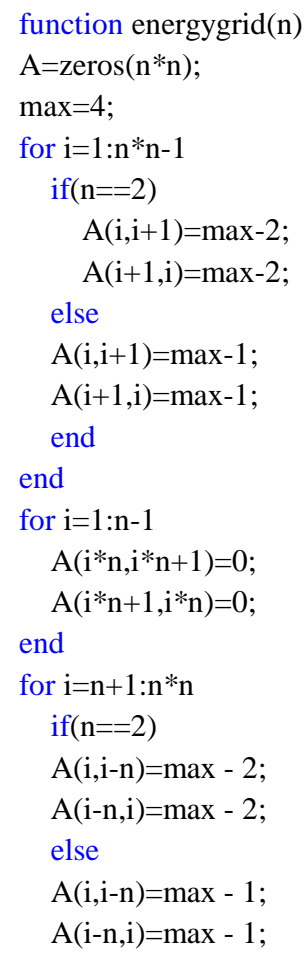




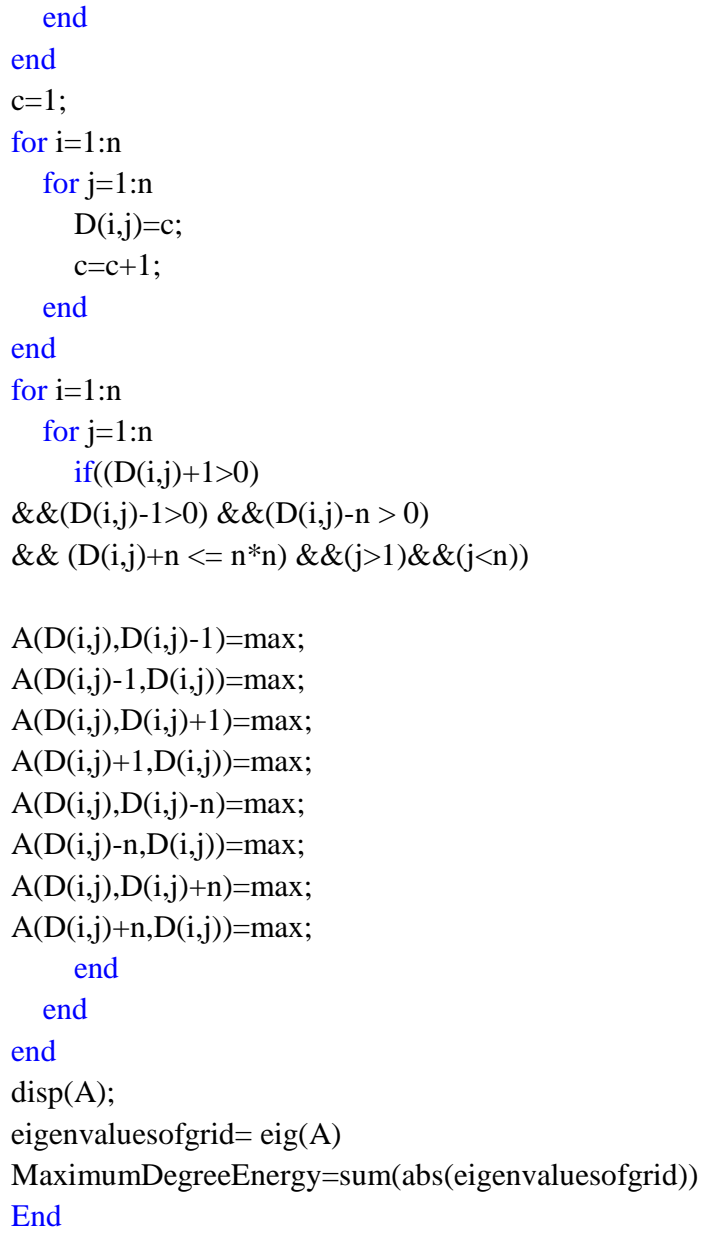

\subsection{Cylinder C $(n, n)$}

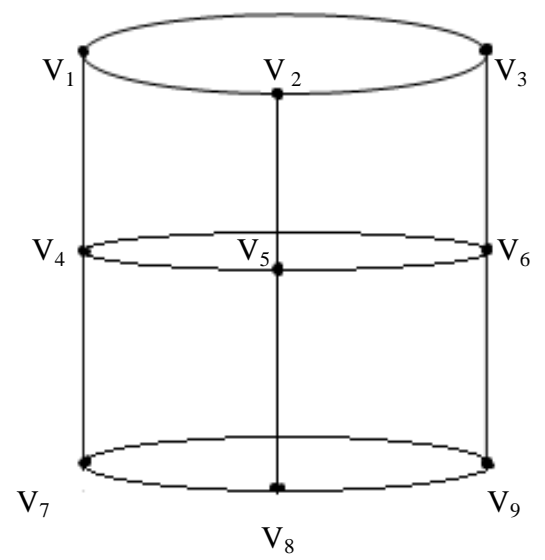

Figure 2: Cylinder $(3,3)$

The maximum degree adjacency matrix of $3 \times 3$ cylinder is given by

$$
\mathrm{C}=\left(\begin{array}{lll}
0 & 4 & 4 \\
4 & 0 & 4 \\
4 & 4 & 0
\end{array}\right), \mathrm{D}=\left(\begin{array}{lll}
4 & 0 & 0 \\
0 & 4 & 0 \\
0 & 0 & 4
\end{array}\right) \text { and }
$$

$\mathrm{O}$ is the zero matrix of size $3 \times 3$.

In general the maximum degree adjacency matrix of cylinder $\mathrm{C}(\mathrm{n}, \mathrm{n})$ for $n \geq 3$ can be written as

$$
\mathrm{A}=\left(\begin{array}{lllllllll}
B & D & O & . & . & . & . & O & O \\
D & C & D & O & . & . & . & O & O \\
O & D & C & D & O & . & . & . & . \\
\cdot & O & D & C & D & O & . & . & . \\
\cdot & \cdot & . & . & . & . & . & . & . \\
\cdot & \cdot & . & . & . & . & . & . & . \\
\cdot & . & . & . & . & D & C & D & O \\
O & O & . & . & . & O & D & C & D \\
O & O & . & . & . & . & O & D & B
\end{array}\right) \text { where }
$$$$
\mathrm{B}=\left(\begin{array}{lllllllll}
0 & 3 & 0 & . & . & . & . & 0 & 3 \\
3 & 0 & 3 & 0 & . & . & . & 0 & 0 \\
0 & 3 & 0 & 3 & 0 & . & . & . & . \\
. & 0 & 3 & 0 & 3 & 0 & . & . & . \\
. & . & . & . & . & . & . & . & . \\
. & . & . & . & . & . & . & . & . \\
. & . & . & . & . & 3 & 0 & 3 & 0 \\
0 & 0 & . & . & . & 0 & 3 & 0 & 3 \\
3 & 0 & . & . & . & . & 0 & 3 & 0
\end{array}\right)
$$$$
\mathrm{C}=\left(\begin{array}{ccccccccc}
0 & 4 & 0 & . & . & . & . & 0 & 4 \\
4 & 0 & 4 & 0 & . & . & . & 0 & 0 \\
0 & 4 & 0 & 4 & 0 & . & . & . & . \\
. & 0 & 4 & 0 & 4 & 0 & . & . & . \\
. & . & . & . & . & . & . & . & . \\
. & . & . & . & . & . & . & . & . \\
. & . & . & . & 0 & 4 & 0 & 4 & 0 \\
0 & 0 & . & . & . & 0 & 4 & 0 & 4 \\
4 & 0 & . & . & . & . & 0 & 4 & 0
\end{array}\right)
$$

$\mathrm{A}=\left(\begin{array}{lll}B & D & O \\ D & C & D \\ O & D & B\end{array}\right)$ where $\mathrm{B}=\left(\begin{array}{lll}0 & 3 & 3 \\ 3 & 0 & 3 \\ 3 & 3 & 0\end{array}\right)$, 
$\mathrm{D}=\left(\begin{array}{ccccccccc}4 & 0 & 0 & . & . & . & . & 0 & 0 \\ 0 & 4 & 0 & 0 & . & . & . & 0 & 0 \\ 0 & 0 & 4 & 0 & 0 & . & . & . & . \\ . & 0 & 0 & 4 & 0 & 0 & . & . & . \\ . & . & . & . & . & . & . & . & . \\ . & . & . & . & . & . & . & . & . \\ . & . & . & . & 0 & 0 & 4 & 0 & 0 \\ 0 & 0 & . & . & . & 0 & 0 & 4 & 0 \\ 0 & 0 & . & . & . & . & 0 & 0 & 4\end{array}\right)$

and $\mathrm{O}$ is the zero matrix of size $n \times n$.

The following MATLAB program generates the maximum degree energy of Cylinder $\mathrm{C}(n, n)$ for $n \geq 3$.

function energycylinder(n)

$\mathrm{A}=\mathrm{zeros}(\mathrm{n} * \mathrm{n})$;

$\max =4$;

for $\mathrm{i}=1: \mathrm{n} * \mathrm{n}-1$

$\mathrm{A}(\mathrm{i}, \mathrm{i}+1)=\max -1$;

$\mathrm{A}(\mathrm{i}+1, \mathrm{i})=\max -1$;

end

for $\mathrm{i}=1: \mathrm{n}-1$

$\mathrm{A}(\mathrm{i} * \mathrm{n}, \mathrm{i} * \mathrm{n}+1)=0$;

$\mathrm{A}(\mathrm{i} * \mathrm{n}+1, \mathrm{i} * \mathrm{n})=0$

end

for $\mathrm{i}=1: \mathrm{n}$

$\mathrm{A}(\mathrm{i} * \mathrm{n}-(\mathrm{n}-1), \mathrm{i} * \mathrm{n})=\max -1$;

$\mathrm{A}(\mathrm{i} * \mathrm{n}, \mathrm{i} * \mathrm{n}-(\mathrm{n}-1))=\max -1$;

end

for $i=n+1: n * n$

$\mathrm{A}(\mathrm{i}, \mathrm{i}-\mathrm{n})=\max$;

$\mathrm{A}(\mathrm{i}-\mathrm{n}, \mathrm{i})=\max$;

end

$\mathrm{c}=1$;

for $\mathrm{i}=1: \mathrm{n}$

for $\mathrm{j}=1: \mathrm{n}$

$\mathrm{D}(\mathrm{i}, \mathrm{j})=\mathrm{c}$;

$\mathrm{c}=\mathrm{c}+1$;

end

end

for $\mathrm{i}=1: \mathrm{n}$

for $\mathrm{j}=1: n$

if $((\mathrm{D}(\mathrm{i}, \mathrm{j})+1>0)$

$\& \&(\mathrm{D}(\mathrm{i}, \mathrm{j})-1>0) \& \&(\mathrm{D}(\mathrm{i}, \mathrm{j})-\mathrm{n}>0)$

$\& \&(D(i, j)+n<=n * n) \& \&(j>1) \& \&(j<n))$

$A(D(i, j), D(i, j)-1)=\max$;

$A(D(i, j), D(i, j)+1)=\max ;$

$A(D(i, j), D(i, j)-n)=\max$;

$A(D(i, j), D(i, j)+n)=\max$; end

end

end

for $\mathrm{i}=1: \mathrm{n}$

for $\mathrm{j}=1: n$ if $((D(i, j)-n>0) \& \&(D(i, j)+n<=n * n)$

$\& \&(\mathrm{j}==1)) \operatorname{disp}(\mathrm{D}(\mathrm{i}, \mathrm{j}))$;

$A(D(i, j), D(i, j)+1)=\max$;

$A(D(i, j), D(i, j)+(n-1))=\max$

$A(D(i, j), D(i, j)-n)=\max$;

$A(D(i, j), D(i, j)+n)=\max$;

end

end

end

for $\mathrm{i}=1: \mathrm{n}$

for $\mathrm{j}=1: \mathrm{n}$

if $((D(i, j)-n>0) \& \&(D(i, j)+n<=n * n) \& \&(j==n))$

$\operatorname{disp}(\mathrm{D}(\mathrm{i}, \mathrm{j}))$

$A(D(i, j), D(i, j)-1)=\max$;

$A(D(i, j), D(i, j)-(n-1))=\max$;

$A(D(i, j), D(i, j)-n)=\max$;

$A(D(i, j), D(i, j)+n)=\max$;

end

end

end

$\mathrm{A} \% \operatorname{disp}(\mathrm{A})$

eigenvaluesofcylinder=eig(A);

MaximumDegreeEnergy=sum(abs(eigenvaluesofcylinder) disp(eigenvaluesofcylinder);

disp(Energy);

end

\subsection{Torus $T(n, n)$}

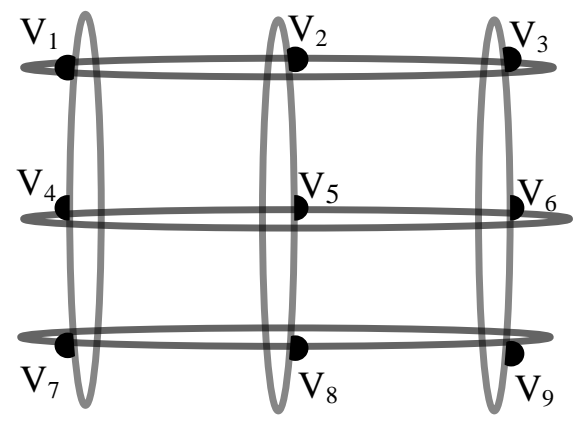

Figure 3: Torus $(3,3)$

The maximum degree adjacency matrix of $3 \times 3$ torus is given by

$$
\begin{gathered}
\mathrm{A}=\left(\begin{array}{lll}
B & C & C \\
C & B & C \\
C & C & B
\end{array}\right) \text { where } \\
\mathrm{B}=\left(\begin{array}{lll}
0 & 4 & 4 \\
4 & 0 & 4 \\
4 & 4 & 0
\end{array}\right), \quad \mathrm{C}=\left(\begin{array}{lll}
4 & 0 & 0 \\
0 & 4 & 0 \\
0 & 0 & 4
\end{array}\right)
\end{gathered}
$$

and $\mathrm{O}$ is the zero matrix of size $3 \times 3$.

In general the maximum degree adjacency matrix of Torus $\mathrm{T}$ (n, $\mathrm{n}$ ) for $n \geq 3$ can be written as 
$\mathrm{A}=\left(\begin{array}{lllllllll}B & C & O & . & . & . & . & O & C \\ C & B & C & O & . & . & . & O & O \\ O & C & B & C & O & . & . & . & . \\ \cdot & O & C & B & C & O & . & . & . \\ \cdot & . & . & . & . & . & . & . & . \\ . & . & . & . & . & . & . & . & . \\ . & . & . & . & O & C & B & C & O \\ O & O & . & . & . & O & C & B & C \\ C & O & . & . & . & . & O & C & B\end{array}\right)$ where

$$
\mathrm{B}=\left(\begin{array}{ccccccccc}
4 & 0 & 0 & . & . & . & . & 0 & 4 \\
0 & 4 & 0 & 0 & . & . & . & 0 & 0 \\
0 & 0 & 4 & 0 & 0 & . & . & . & . \\
. & 0 & 0 & 4 & 0 & 0 & . & . & . \\
. & . & . & . & . & . & . & . & . \\
. & . & . & . & . & . & . & . & . \\
. & . & . & . & 0 & 0 & 4 & 0 & 0 \\
0 & 0 & . & . & . & 0 & 0 & 4 & 0 \\
4 & 0 & . & . & . & . & 0 & 0 & 4
\end{array}\right)
$$$$
\mathrm{C}=\left(\begin{array}{ccccccccc}
0 & 4 & 0 & . & . & . & . & 0 & 0 \\
4 & 0 & 4 & 0 & . & . & . & 0 & 0 \\
0 & 4 & 0 & 4 & 0 & . & . & . & . \\
. & 0 & 4 & 0 & 4 & 0 & . & . & . \\
. & . & . & . & . & . & . & . & . \\
. & . & . & . & . & . & . & . & . \\
. & . & . & . & 0 & 4 & 0 & 4 & 0 \\
0 & 0 & . & . & . & 0 & 4 & 0 & 4 \\
0 & 0 & . & . & . & . & 0 & 4 & 0
\end{array}\right)
$$

and $\mathrm{O}$ is the zero matrix of size $n \times n$.

The following MATLAB program generates the maximum degree energy of Torus $T(n, n)$

function energytorus(n)

$\mathrm{A}=\mathrm{zeros}(\mathrm{n} * \mathrm{n})$;

$\max =4$;

for $i=1: n * n-1$

$\mathrm{A}(\mathrm{i}, \mathrm{i}+1)=\max$;

$\mathrm{A}(\mathrm{i}+1, \mathrm{i})=\max$; end

for $\mathrm{i}=1: \mathrm{n}-1$

$\mathrm{A}(\mathrm{i} * \mathrm{n}, \mathrm{i} * \mathrm{n}+1)=0$;

$\mathrm{A}(\mathrm{i} * \mathrm{n}+1, \mathrm{i} * \mathrm{n})=0$;

end

for $i=1: n$

$\mathrm{A}(\mathrm{i} * \mathrm{n}-(\mathrm{n}-1), \mathrm{i} * \mathrm{n})=\max ;$

$\mathrm{A}(\mathrm{i} * \mathrm{n}, \mathrm{i} * \mathrm{n}-(\mathrm{n}-1))=\max$;

end

for $\mathrm{i}=\mathrm{n}+1: \mathrm{n} * \mathrm{n}$

$\mathrm{A}(\mathrm{i}, \mathrm{i}-\mathrm{n})=\max$;

$\mathrm{A}(\mathrm{i}-\mathrm{n}, \mathrm{i})=\max$;

end

for $\mathrm{i}=\mathrm{n} *(\mathrm{n}-1)+1: \mathrm{n} * \mathrm{n}$

$\mathrm{A}(\mathrm{i}, \mathrm{i}-\mathrm{n} *(\mathrm{n}-1))=\max$;

$\mathrm{A}(\mathrm{i}-\mathrm{n} *(\mathrm{n}-1), \mathrm{i})=\max$;

end

Aeigenvaluesoftorus $=\operatorname{eig}(\mathrm{A})$

MaximumDegreeEnergy=sum(abs(eigenvaluesoftorus))

End

\subsection{Extended grid EX $(n \times n)$}

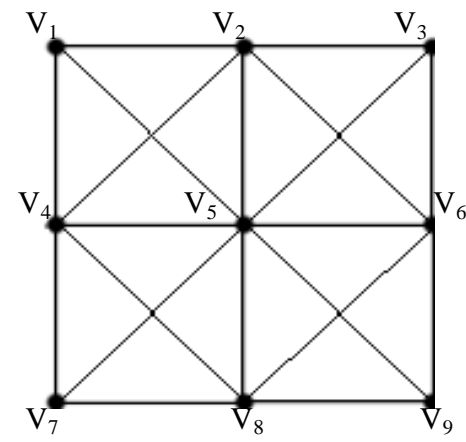

Figure 4: Extended grid $(3,3)$

The maximum degree adjacency matrix of Extended grid $\mathrm{EX}(3 \times 3)$ is given by

$$
\begin{aligned}
\mathrm{A} & =\left(\begin{array}{ccc}
B & D & O \\
D^{T} & C & D^{T} \\
O & D & B
\end{array}\right) \text { where, } \mathrm{B}=\left(\begin{array}{lll}
0 & 5 & 0 \\
5 & 0 & 5 \\
0 & 5 & 0
\end{array}\right), \\
\mathrm{C} & =\left(\begin{array}{lll}
0 & 8 & 0 \\
8 & 0 & 8 \\
0 & 8 & 0
\end{array}\right), \mathrm{D}=\left(\begin{array}{lll}
5 & 8 & 0 \\
5 & 8 & 5 \\
0 & 8 & 5
\end{array}\right)
\end{aligned}
$$

and $\mathrm{O}$ is the zero matrix of size $3 \times 3$.

In general the maximum degree adjacency matrix of $\mathrm{EX}(\mathrm{n}, \mathrm{n})$ for $n \geq 4$ can be written as 


$$
\mathrm{A}=\left(\begin{array}{ccccccccc}
B & D & O & . & . & . & . & O & 0 \\
D^{T} & C & E & O & . & . & . & O & 0 \\
O & E & C & E & O & . & . & . & . \\
\cdot & O & E & C & E & O & . & . & . \\
\cdot & . & . & . & . & . & . & . & . \\
\cdot & . & . & . & . & . & . & . & . \\
\cdot & . & . & . & O & E & C & E & 0 \\
O & 0 & . & . & . & O & E & C & D^{T} \\
O & 0 & . & . & . & . & O & D & B
\end{array}\right)
$$$$
\mathrm{B}=\left(\begin{array}{ccccccccc}
0 & 5 & 0 & . & . & . & . & 0 & 0 \\
5 & 0 & 5 & 0 & . & . & . & 0 & 0 \\
0 & 5 & 0 & 5 & 0 & . & . & . & . \\
. & 0 & 5 & 0 & 5 & 0 & . & . & . \\
. & . & . & . & . & . & . & . & . \\
. & . & . & . & . & . & . & . & . \\
. & . & . & . & 0 & 5 & 0 & 5 & 0 \\
0 & 0 & . & . & . & 0 & 5 & 0 & 5 \\
0 & 0 & . & . & . & . & 0 & 5 & 0
\end{array}\right)
$$$$
\mathrm{C}=\left(\begin{array}{ccccccccc}
0 & 8 & 0 & 0 & . & . & . & 0 & 0 \\
8 & 0 & 8 & 0 & 0 & . & . & 0 & 0 \\
0 & 8 & 0 & 8 & 0 & 0 & . & . & . \\
. & 0 & 8 & 0 & 8 & 0 & 0 & . & . \\
. & . & . & . & . & . & . & . & . \\
. & . & . & . & . & . & . & . & . \\
. & . & . & . & . & 8 & 0 & 8 & 0 \\
0 & 0 & . & . & . & 0 & 8 & 0 & 8 \\
0 & 0 & . & . & . & . & 0 & 8 & 0
\end{array}\right)
$$

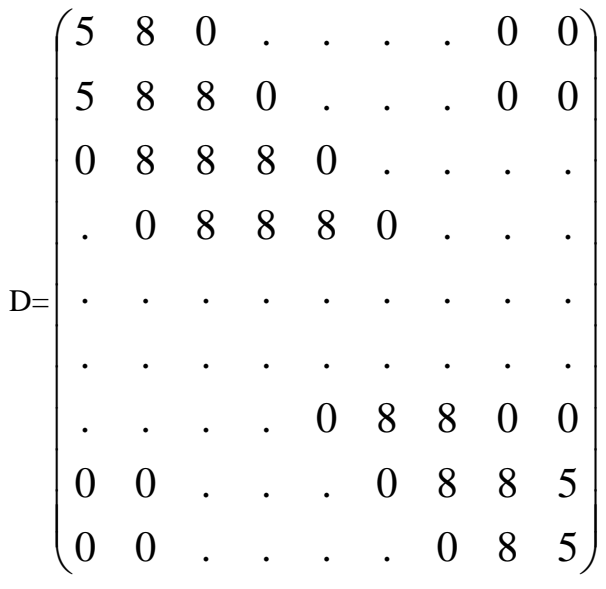

$\mathrm{E}=\left(\begin{array}{ccccccccc}5 & 8 & 0 & . & . & . & . & 0 & 0 \\ 8 & 8 & 8 & 0 & . & . & . & 0 & 0 \\ 0 & 8 & 8 & 8 & 0 & . & . & . & . \\ . & 0 & 8 & 8 & 8 & 0 & . & . & . \\ . & . & . & . & . & . & . & . & . \\ . & . & . & . & . & . & . & . & . \\ . & . & . & . & 0 & 8 & 8 & 8 & 0 \\ 0 & 0 & . & . & . & 0 & 8 & 8 & 8 \\ 0 & 0 & . & . & . & . & 0 & 8 & 5\end{array}\right)$

$\mathrm{O}$ is the zero matrix of size $\mathrm{n} \times \mathrm{n}$.

The following MATLAB program generates the maximum degree energy of Extended Grid $(n, n)$

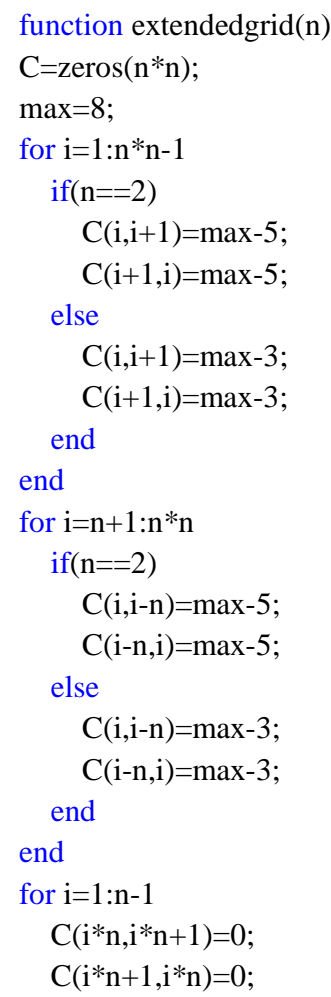




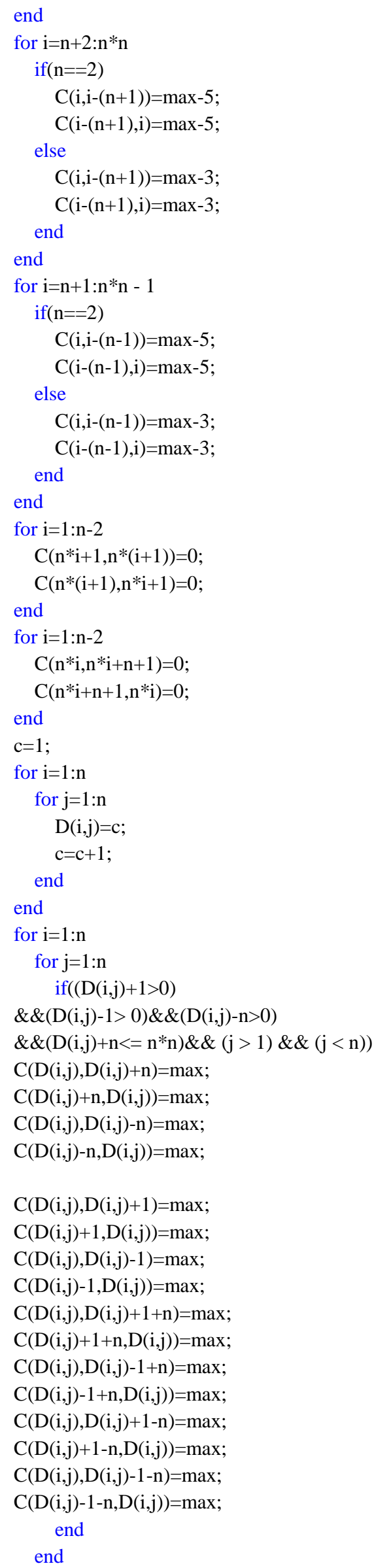

end

$\mathrm{C} \% \operatorname{disp}(\mathrm{C})$

eigenvaluesofgrid $=\operatorname{eig}(\mathrm{C})$

MaximumDegreeEnergyofextended_grid=sum(abs (eigenvaluesofgrid))

End

\section{REFERENCES}

[1] C.Adiga, A.Bayad, I.Gutman, S.A.Srinivas, The minimum covering energy of a graph,Kragujevac J. Sci. 34 (2012), 39-56.

[2] C. Adiga and M. Smitha, On Maximum degree energy of a graph, Int.J.Contemp.Math.sciences, Vol.4 (2009), NO.8, 385-396.

[3] Bharati Rajan, Sudeep Stephen, Albert William, Cyriac Grigorous, On Laplacian Energy of Certain Mesh Derived Networks, International Journal of Computer Applications, Vol. 55- No.11, (2012).

[4] D.Cvetković, I. Gutman (eds.),Applications of Graph Spectra (Mathematical Institution, Belgrade,2009)

[5] D. Cvetković, I. Gutman (eds.) Selected Topics on Applications of Graph Spectra, (Mathematical Institute Belgrade,2011)

[6] I. Gutman, The energy of a graph.Ber. Math-Statist. Sekt. Forschungsz.Graz 103, 1-22 (1978).

[7] I. Gutman, X. Li, J.Zhang, in Graph Energy, ed. by M.Dehmer, F.Emmert - Streib. Analysis of Complex Networks. From Biology to Linguistics (Wiley VCH, Weinheim, (2009), pp. 145174.

[8] I. Gutman, O.E. Polansky, Mathematical Concepts in Organic Chemistry (Springer, Berlin,1986).

[9] I. Gutman, in The energy of a graph: Old and New Results,ed.by A. Betten, A. Kohnert, R. Laue, A. Wassermann. Algebraic Combinatorics and applications (Springer, Berlin, 2001), pp. 196 - 211.

[10] A.Graovac,I.Gutman, N.Trinajsti_c,Topological Approach to the Chemistry of Conjugated molecules (Springer, Berlin, 1977).

[11] Huiqing Liu, Mei Lu and Feng Tian, Some upper bounds for the energy of graph Journal of Mathematical Chemistry, Vol. 41, No.1, (2007).

[12] B.J.McClelland, Properties of the latent roots of a matrix: The estimation of $\pi$-electron energies. J.Chem. phys.54, 640 - 643 (1971).

[13] M.R.Rajesh Kanna, B. N. Dharmendra, and G. Sridhara, Minimum Dominating Energy of a Graph. International Journal of Pure and Applied Mathematics, 85, No. 4 (2013) 707-718. 